\title{
Space Leakage of Power from the Slotline
}

\author{
Jan Zehentner, Jan Machac, and Petr Lorenz \\ Czech Technical University, Technicka 2, 16627 Prague 6, Czech Republic
}

\begin{abstract}
In this paper we present the comprehensive dispersion characteristics of the slotline on thin and low permittivity substrate with a wide slotwidth comprising the bound and space leaky waves with even and odd electric field symmetry. The final design and measurement of the slotline antenna radiation are based on our analysis of this slotline. The antenna and its feeding circuit are designed assuming only the first odd space leaky wave radiation. Measured and calculated return losses and radiation patterns of the antenna are shown. Remarks on the applicability of the antenna conclude this treatise.
\end{abstract}

\section{INTRODUCTION}

In recent years many research groups round the world have been investigating printed-circuit lines and the passive elements derived from them. They are working either on circuit elements with distributed parameters, including line discontinuities, or on various kinds of radiators that can be used as single or array antennas. We have studied uniplanar transmission lines made on a dielectric substrate operating in the microwave and millimeterwave ranges. Recently we have focused our interest on the space leakage of power. This programme has led us to investigate space leaky waves on the slotline and ways of using them to construct a slotline antenna. The aim of this paper is the presentation of our new findings in the design, manufacture and measurement of the slotline radiating the space leaky wave.

We presented in [1] an overview and classification of the dominant and higher order bound waves and the space leaky waves of the 1st and 2nd class with odd and even electric field symmetry within the slot. Substrate permittivity 10.8 was chosen in order to make a clear distinction between particular waves. Now, in order to verify them experimentally at frequencies up to $12.5 \mathrm{GHz}$, where measuring equipment is easy accessible, we had to recalculate the dispersion characteristics on a low permittivity substrate. Unfortumately, in this case the phase constants of all the relevant waves are compressed in the close vicinity of the free space constant $\mathrm{k}_{0}$. Consequently, the choice of the feeder providing excitation of the desired mode is very important. In addition to low permittivity, thin substrate also had to be applied in order to observe the space leakage.

The experiment revealed some new factors influencing the field behaviour that were not known up to now and had not been accounted for in the line analysis. This concerns a rise in the residual standing waves and their impact on the radiation pattern. An antenna radiating the 1st space leaky wave of the first class has a relatively low gain, a wide beam width in the E plane, a narrower beam width in the $\mathrm{H}$ plane, and moderate frequency scan ability. A possible application for this may be in the mm-wave range, where it has acceptably small dimensions and the array design is envisaged. A conductor backed slotline antenna operating with the leaky wave is potentially suitable for placing on the bulk surface.

\section{SPace Leaky WaVe ON The SLOtLINE}

We investigated the space leaky waves on the slotline, the cross-section of which is shown in the inset of Fig. 2, by means of the method of moments in the spectral domain. The path of integration in the complex plane of the spectral variable embraces the poles related with the surface waves on a grounded dielectric slab and the branch cut with the branch point. Since cheap plexiglass sheets are available and also measurement on an enlarged model of the slotline at frequencies lower than $12.5 \mathrm{GHz}$ is easier than in the mm-wave range, we show in Fig. 1 the dispersion characteristics of the slotline with a substrate thickness of $1.2 \mathrm{~mm}$, permittivity 2.6, with a slot width of $60 \mathrm{~mm}$.

According to the transversal electric field component on the substrate surface within the slot we distinguish waves with even and odd symmetry with respect to a plane positioned along the line axis and perpendicular to the substrate. On the given slotline, dependent on the source, the dominant bound wave with even symmetry, the 1st higher order bound wave with odd symmetry, the 1st space leaky wave of the first class with odd symmetry, the 3 rd space leaky wave of the 2 nd class with odd symmetry, and the 2nd space leaky wave of the 2nd class with even symmetry may propagate. The 2 nd higher order bound wave, the 2nd space leaky wave of the first class, both with even symmetry, and also all surface leaky waves, may occur at frequencies higher than $15 \mathrm{GHz}$.

Let us now observe radiation of the 1st space leaky wave of the first class. For the sake of better readibility, only the normalized phase and leakage constants of present interest are redrawn from Fig. 1 into Fig. 2. 


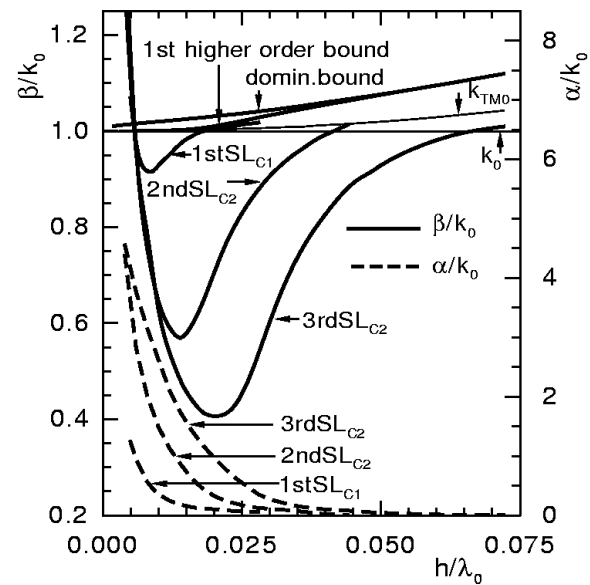

Fig. 1. Normalized phase $\beta / \mathrm{k}_{0}$ and leakage $\alpha / \mathrm{k}_{0}$ constant of the slotline with $\mathrm{w}=60 \mathrm{~mm}, \mathrm{~h}=1.2 \mathrm{~mm}, \varepsilon_{\mathrm{r}}=2.6$ depending on the normalized frequency $\mathrm{h} / \lambda_{0}$.

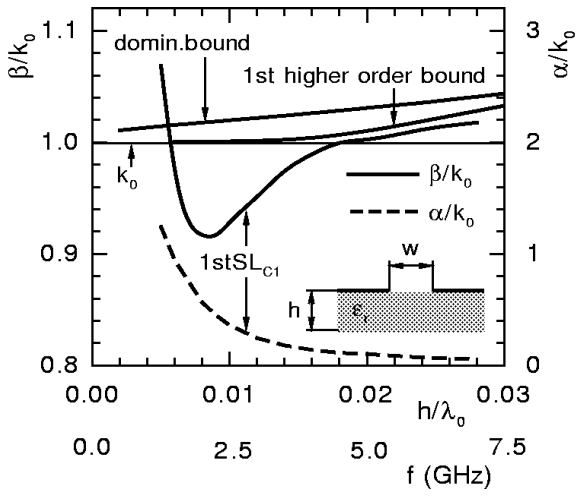

Fig. 2. Normalized phase and leakage constant of the 1st space leaky wave of first class on the slotline from Fig. 1 up to 7.5 GHz.

A microstrip patch placed axially along the center line of the slotline on the opposite side of the substrate provided excitation of this wave, as in [2]. We confirmed that this wave dominated in the excited spectrum. Mapping of the field by a small probe movable across and along the slot provided evidence for this statement. The transversal electric field component $\mathrm{E}_{\mathrm{x}}$ exhibited a typical record with maxima at the edges of both conductors and with zero in the middle of the slot as is shown in Fig. 3. The field was scanned at three distances from the source differing from each other by $100 \mathrm{~mm}$. The measured decrease of the amplitude along the line reasonably agreed with the calculated decrease, when only the leakage constant of the 1st leaky wave was accounted for as Fig. 4 documents. We also determined the wavelength from the measured nodes of the standing waves produced by a short, that is movable along the slot. With an error less than $3 \%$, it compares with the wavelength calculated from the phase
Fig. 3. Measured and calculated transversal electric field component $E_{x}$ across the slot of the line from Fig. 2 at the frequency $3.25 \mathrm{GHz}$ and at the distance $130 \mathrm{~mm}$ from the feeding microstrip patch.

Fig. 4. Measured and calculated decay of the transversal electric field component $\mathrm{E}_{\mathrm{x}}$ along the slotline from Fig. 2 when the position of the probe is taken from the edge of the feeding microstrip patch.

constant of the 1st space leaky wave. The wavelength check may be influenced by the possible presence of the 1st higher order bound wave in the spectrum. The chosen feeder is unable to excite the dominant bound wave, the 2nd and 3rd space leaky wave of the second class, since their field and the feeder field distribution are not in conformity with each other. Unfortunately, the field of the 1 st higher order bound wave resembles the field of the 1 st space leaky wave of the first class, therefore these two waves can excite simultaneously. Their phase constants are close to each other, and it has not yet been possible to separate them satisfactory. The two phase constants differ by $7.3,3.6$, and $1.4 \%$ at $2.5,3.25$, and $4.0 \mathrm{GHz}$, respectively.

From our measurement we concluded that the 1st space leaky wave of the first class dominates, and the 1st higher order bound wave plays a minor part in the total spectrum on the slotline under investigation, as was observed in [2].

\section{RADIATION FROM THE SLOTLINE}

We acknowledge in [1] the excitation and phase constant of the 1st space leaky wave of the first class on the slotline. To excite this wave in the current experiment, the microstrip stub was placed on the longitudinal slotline axis on the conductorless side of the substrate. The stub dimensions were optimized for given slotline cross-section dimensions at $3.1 \mathrm{GHz}$ by means of the Zeland IE3D simulator. The calculated and measured return losses are shown in Fig. 5. The applied model of the source in the

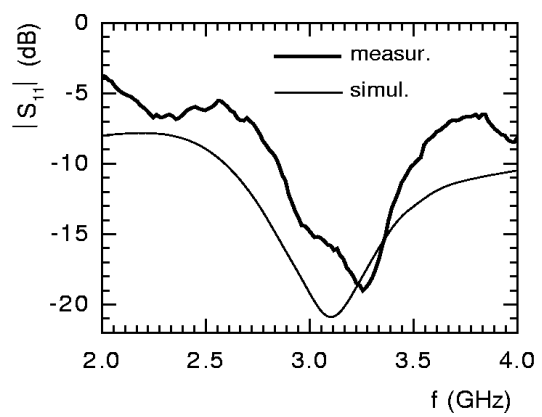

Fig. 5. Measured and simulated return loss of the slotline antenna with $\mathrm{w}=60 \mathrm{~mm}, \mathrm{~h}=1.2 \mathrm{~mm}, \varepsilon_{\mathrm{r}}=2.6$. 
simulator was responsible for the slight frequency displacement of the best match. Junction resistor between the inner conductor of the coaxial connector and the microstrip patch, losses in the substrate, in the aluminium metallization, and in the adhesive, not accounted for in the simulation, produced worse return losses than their predicted values. The length of the slotline was determined by the leakage constant of the desired 1st space leaky wave, i. e., by the distance where its amplitude decreased practically to zero. Beyond this point a short narrow slotline was added at which this wave and also the 1st higher order bound wave are not allowed to propagate.

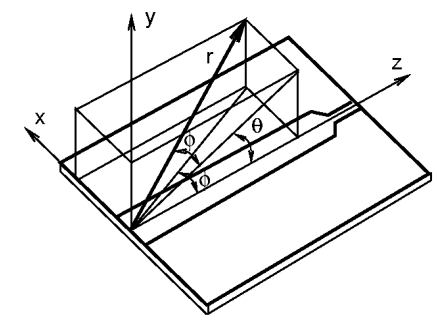

Fig. 6. Co-ordinates and the slotline antenna arrangement.

The orientation of the co-ordinates with respect to the slotline is sketched in Fig. 6. The measured and simulated radiation pattern $E_{\theta}(\theta)$ of the slotline antenna in the vertical plane $\phi=0$ and the calculated radiation pattern of an ideal travelling-wave line source [3] for $2.5 \mathrm{GHz}$ are shown in Fig. 7. All three traces agree well. A rise in the frequency resulted in greater disagreement between the ideal travelling-wave line source radiation pattern and the measured and simulated patterns, as shown in Fig. 8, valid for $3.25 \mathrm{GHz}$. The simple field distribution assumed in the slot of the ideal travelling-wave source is not an adequate model of the actual field distribution of the 1st space leaky wave in the wide slot at higher frequencies. This fact is responsible for the discrepancy of the traces in question. Nevertheless, up to $4 \mathrm{GHz}$, simulated and measured radiation patterns compare well.

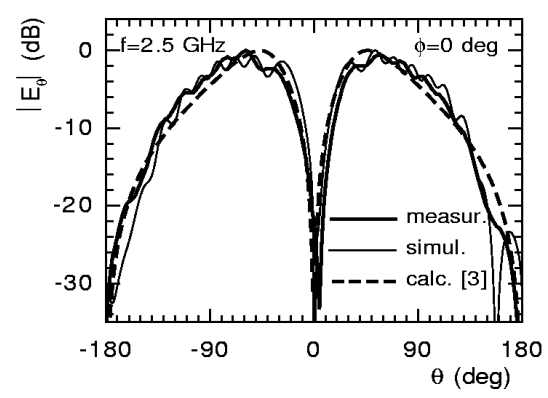

Fig. 7. Measured, simulated and calculated [3] $\mathrm{E}_{\theta}(\theta)$ radiation patterns of the leaky wave slotline antenna in the plane $\phi=0$ degree at $2.5 \mathrm{GHz}$.

Ripples in the radiation pattern arose due to undesired standing waves that occurred in the metallized area. These practically disappeared at lower frequencies, e. g., at 2.5 $\mathrm{GHz}$, and arose with frequency increase, e. g., at $4.0 \mathrm{GHz}$. Our current effort is to remove them entirely in the whole operation frequency band.

Radiation pattern $E_{\theta}(\phi)$ at $3.25 \mathrm{GHz}$ in the plane of the highest radiation inclined by 28 degrees from the horizontal plane is plotted in Fig. 9. The main beam width in this plane is narrower than in the $\phi=0$ plane.

The main beam direction inclined with the frequency. At 2.5, 3.0, 3.5 and $4.0 \mathrm{GHz}$ its inclination from the horizontal plane was 36.3, 33.0, 26.1 and 22.7 degrees, respectively.

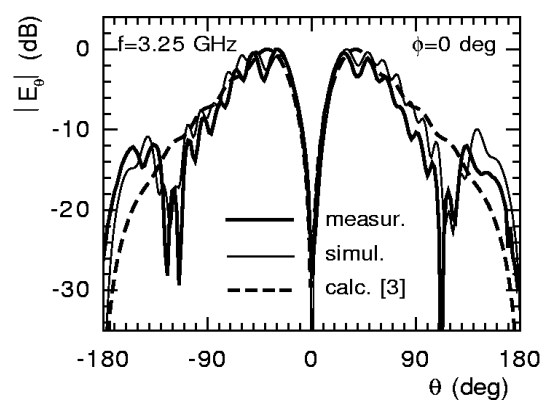

Fig. 8. Measured, simulated and calculated [3] $\mathrm{E}_{\theta}(\theta)$ radiation patterns of the leaky wave slotline antenna in the plane $\phi=0$ degree at $3.25 \mathrm{GHz}$.

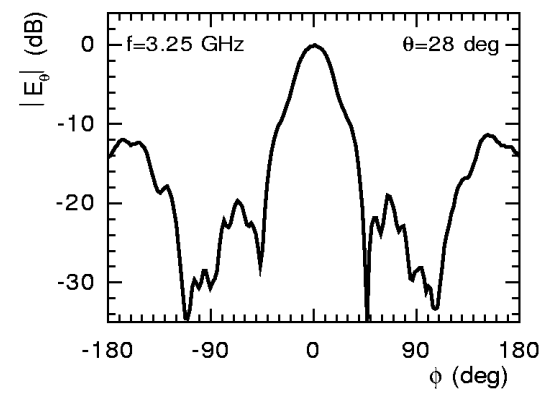

Fig. 9. Measured radiation pattern $\mathrm{E}_{\theta}(\phi)$ in the plane $\theta=28$ degree at $3.25 \mathrm{GHz}$.

In addition we modified the cross-section of the slotline. The substrate was totally metallized with the exception of the slot and the microstrip patch area. The latter was covered by metallization underlaid by the same thin dielectric substrate. Now the main beam width became wider and the antenna radiated only to the half-space with $-10 \mathrm{~dB}$ side lobes. 


\section{CONCLUSIONS}

The slotline with a wide slot on a thin and low permittivity substrate was investigated. Emphasis was put on radiation of power by the space leaky wave. The dispersion equation was solved for the propagation constant of particular waves. It turned out that at low frequencies the wave spectrum is clear and includes only the dominant and 1st higher order bound wave, the 1st space leaky wave of the first class, and the 2nd and 3rd space leaky wave of the second class. A properly designed source can excite the desired wave, which in our case was the 1 st space leaky wave of the first class. Due to the resemblance of its field to the 1 st higher order bound wave field distribution, this wave may also occur. We hope to eliminate this bound wave by careful adjustment of the slotwidth.

We excited the slotline through the microstrip patch on the opposite side of the substrate with much better efficiency than Sheen and Lin achieved in [2]. Similarly we believe we can achieve CPW excitation that is totally compatible with a uniplanar concept of the structure.

Field mapping across and along the slot confirmed the presence and domination of the 1st space leaky wave of the first class. The phase and leakage constants determined by measurement of the attenuation of the propagating wave and measurement of the nodes of the standing waves compared well with calculated values. The slotline radiated two main beams, each on the opposite side of the substrate. The beam width in the E plane is greater than in the $\mathrm{H}$ plane. The backward lobes are due to the open area of the feeder junction. Undesired standing waves, which occurred at higher frequencies, produced ripples in the radiation patterns. Frequency scanning sensitivity 7.7 $\mathrm{deg} / \mathrm{GHz}$ in the $2.5-4.0 \mathrm{GHz}$ range was more than seven times greater than $1 \mathrm{deg} / \mathrm{GHz}$ within the $14-18 \mathrm{GHz}$ range in [2].

Metallization on the back side and on the lateral edges of the substrate ensured the radiation of only one main beam. We will follow up this concept in future investigations. A radiating leaky wave slotline antenna could become a counterpart to the microstrip leaky wave antenna [4]. Its advantage is that on the upper surface of the substrate only the slot is etched, and this does not much damage the metal surface of the bulk body.

\section{ACKNOWLEDGEMENT}

This work has been supported by the Grant Agency of the Czech Republic under project 102/00/0025 „Planar circuits for mw and mmw technology“ and by research programmes J04/98 212300014 „Information and Communication Technology" and MSM 216200031 „Intensive Numerical Calculations and Parallel Programming“.

\section{REFERENCES}

[1] J. Zehentner, J. Machac, "New Space Leaky Wave Spectrum Components on the Slotline," Conference Proceedings of the 30th EuMC, vol. 2, pp. 190-193, June 2000 Paris.

[2] J.-W. Sheen, Y.-D. Lin, "Propagation Characteristics of the Slotline First Higher Order Mode", IEEE Trans. Microwave Theory and Tech., vol. MTT-46, no. 11, pp. 1774-1781, Nov. 1998.

[3] C. H. Walter, Traveling Wave Antennas, New York: McGraw-Hill,1965.

[4] W. Menzel, "A new traveling-wave antenna in microstrip", Arch. Electr. Uebertrag. Tech., vol. 33, pp. 137-140, Apr. 1979. 